\title{
Successful Treatment of Anal Canal Cancer Metastasis to the Cranial Bones: A Case Report and Literature Review
}

\author{
ILARIA BENEVENTO, FRANCESCA DE FELICE, NADIA BULZONETTI, ROSSELLA CAIAZZO, \\ RAFFAELE CASSESE, DANIELA MUSIO and VINCENZO TOMBOLINI
}

Department of Radiotherapy, Policlinico Umberto I, "Sapienza" University of Rome, Rome, Italy

\begin{abstract}
Single metastasis to the cranial bone represents a very uncommon occurrence that can arise from an anal canal cancer. No cases of cranial bone metastasis from anal canal carcinoma are available in the literature. Herein, we present a case of a unique metastatic lesion to the right parietal bone that occurred after curative chemoradiotherapy of primary squamous cell anal canal carcinoma. The patient received radiotherapy and systemic platinum-based chemotherapy, with optimal local control, high compliance and a well tolerable level of toxicity.
\end{abstract}

Tumors that tend to metastasize to bone structures do not neglect the dome of the skull as a target. Metastases to the cranial bones are relatively rare, accounting for less than $5 \%$ of all secondary bone locations (1). At present, they remain a challenging disease to treat and current guidelines are mainly designed to address improvement in patients' quality of life (QoL) (2). Actually, there is lack of specific guidelines regarding the best therapeutic approach in cranial bone metastasis. Therefore, understanding the role of radiation therapy (RT), chemotherapy (CHT), surgery (S) or combinations of these modalities is of paramount importance, especially in patients with local control of primary tumor.

Herein, we describe a case of cranial bone metastasis of anal canal carcinoma, supporting the proposal that RT is a reasonable primary treatment option in the management of these patients. A comprehensive overview of metastatic tumor to the cranial bones is also provided in order to

This article is freely accessible online.

Correspondence to: Francesca De Felice, Department of Radiotherapy, Policlinico Umberto I "Sapienza" University of Rome, Viale Regina Elena 326, 00161 Rome, Italy. Tel: +39 0649973411, Fax: +39 0649973411, e-mail: fradefelice@hotmail.it

Key Words: Anal canal carcinoma, bone metastasis, calvarial, radiotherapy, chemotherapy, hypofractionated, survival. illustrate the main diagnostic, therapeutic and prognostic aspects of this entity.

All procedures performed were in accordance with the ethical standards of the institutional research committee and comparable with ethical standards. Informed consent was obtained from all individual participants included in the study.

\section{Case Description}

A 69-years old Caucasian female presented to the clinic with vaginal swelling and enlarged left-sided inguinal lymph node in June 2013. Clinical examination revealed a primary anal canal lesion. Biopsy of perianal lesion revealed a squamous cell carcinoma that was human papillomavirus (HPV)negative. Staging computed tomography (CT) of chest, abdomen and pelvis confirmed the metastasis in inguinal lymph node and the vagina tumor invasion, without distant metastasis (cT4, cN2 M0, according to American joint committee on cancer staging 7 th edition).

Due to massive loco-regional extension, before standard definitive chemoradiotherapy (CRT), two cycles of chemotherapy based on Mitomycin C (MMC, $10 \mathrm{mg} / \mathrm{m}^{2}$ day 1) and 5-Fluorouracil (5-FU, continuous infusion $1,000 \mathrm{mg} / \mathrm{m}^{2}$ days 1-5) were planned. Treatment was well tolerated, with high compliance and a relatively good level of toxicity. Patient received the total prescribed RT dose (59.4 Gy, 1.8 Gy per fraction) and the concurrent MMC/5-FU-based CHT during the first and last week of RT treatment. A complete clinical response, confirmed by diagnostic exam, was achieved 6 months after CRT (March 2014). But, in the meantime, the patient developed a painful mass on the right parietal bone. The lesion was fast growing, non-pulsanting and covered by normal cutis. No neurological deficits were noted. A CT of the head revealed a solitary osteolytic hyperdense lesion with contrast enhancement on the right parietal bone measuring $25 \times 16 \mathrm{~mm}$ without dural and brain involvement. A fine needle aspiration biopsy confirmed metastasis from squamous cell carcinoma. After a multidisciplinary discussion, local RT followed by CHT was recommended. RT was delivered with 
a 3D-conformational multiple field technique at a total dose of 45 Gy ( 3 Gy per fraction) to the to the skull lesion, with 6 energy photons. Patient underwent CT scan one-week prior RT. She was treated in a supine position and was immobilized in a thermoplastic shell, fixed to the couch in three places. Bolus was not used. Images were acquired with $3 \mathrm{~mm}$ slice thickness and were obtained from the vault to the base of the skull. Diagnostic CT images were co-registered to the planning CT. Target volume delineation was manually performed and a $5 \mathrm{~mm}$ margin to the gross tumor volume was added.

CHT consisted of up to 4 cycles of three-weekly cisplatin $\left(60 \mathrm{mg} / \mathrm{m}^{2}\right.$ day 1) and 5-FU (continuous infusion $600 \mathrm{mg} / \mathrm{m}^{2}$ days 1-5). Treatment was well tolerated, with no interruptions and severe toxicity. Two months later, in June 2014, follow-up CT imaging showed a complete clinical response (Figure 1). Progression-free survival was achieved until September 2015, when follow-up diagnostic exams confirmed pathological pelvic lymph nodes and vulvar recurrence. Palliative chemotherapy treatment was left to the oncologist's discretion. At December 2017 brain metastasis was documented. Patient's general condition progressively deteriorated and she died in February 2018.

\section{Discussion}

We describe a case of metastatic anal canal cancer to the cranial bone successfully treated with local RT and systemic CHT. After treatment, the patient remained free of relapse for 17 months. This multidisciplinary approach was safe and effective and it was mainly proposed to ensure low morbidity and mortality. Usually, in patients with primary anal canal cancer, the most likely mode of spread to distant sites is through systemic circulation, implying potential systemic tumor dissemination. Based on this rationale, the utility of local RT was explored to reduce the excess of radiation exposure to the peri-lesional brain parenchyma and systemic CHT to control micro-metastasis. The aim was to positively influence survival time, local control and improve patient's QoL.

At present, there are no standardized algorithms to manage cranial bone metastasis. In order to determine the treatment approach, a literature search using PubMed database up to October 2018 was performed using the following combinations of research criteria: "metastasis", "metastatic", "anal canal carcinoma", "cranial", "bone", "skull", "calvarial", "surgery", "radiotherapy", "radiosurgery", and "magnetic resonance image". No articles on cranial bone metastasis from primary anal canal carcinoma were available. To best of our knowledge, our patient represents the first case described in medical literature. Therefore, it was not possible to limit the search to this setting of patients and consequently we enlarged it to include cranial bone metastasis of different origin sites. The search was restricted to English-language papers. English literature lacks large series highlighting cranial bone metastasis. It consists mostly of single case reports $(n=21)$ and a scarce number of case series $(n=13)(2-35)$. Data from each study were tabulated and included authors, year of publication, sample size, primary tumor, cranial bone metastasis characteristics, treatment details and follow-up. A total of 34 studies representing 339 patients were identified. Details are listed in Table I. Reports have described calvarial metastasis from different types of primary cancers, most commonly from kidney $(n=30)$, liver $(n=16)$ and breast $(n=15)$ cancers (It should be noted that $(n)$ indicates the number of patients). Frontal and parietal bones were the most common sites involved $(\mathrm{n}=91)(2-7,9,12,15,17-20$, 22-23, 25-27, 29-32). Clinical presentation mainly depended on the location of the metastatic lesion. Cranial bone metastases were often asymptomatic $(n=13)(3,7-8,13,15$, $19,23,36)$, due to the absence of the innervations of the calvarium (26). Involvement of the outer table of the skull caused superficial focal pain and cosmetic problems $(n=93)$ $(2,4,6,9,12,16,20-22,24,26,28-29,31-32)$. Whereas the infiltration of the inner table was related to neurological symptoms, such as headache and nausea $(n=8)(3-4,20)$. The subsequent extension to eloquent cortex, as well as the impairment of cranial nerves caused neurological deficit (14). Major symptoms, including increased intracranial pressure, meningeal irritation and seizures, occured if the dura and intradural spaces were involved $(n=21)(2-4,20,22$, 28-29). CT scan with bone window was the method of choice to detect bone erosion (22). With CT, calvarial metastasis often presented as an osteolytic lesion. To better delineate lesion boundaries, as well as soft tissue and dural invasion, magnetic resonance imaging (MRI) with noncontrasted sequences was performed in several cases (2, 3739). Cranial bone metastasis showed hypo-intensity on nonenhanced T1-weighted images and a variable appearance on T2-weighted images, in contrast with the hyper-intensity of normal bone marrow $(21,28,31)$. Nowadays, MRI should be considered a standard exam to better classify cranial bone metastasis. Recently, Mitsuya et al. have proposed a classification of skull metastasis based on MRI features (28). A total of 218 metastases were classified with regard to i) location - calvarium or cranial base, ii) distribution within the plane of the cranial bone - confined to one bone or diffuse to another bone spreading across a suture, iii) invasion - in cranial bones only, into the scalp or inward to the dura. Globally, 121 patients have been identified with calvarial bone metastases. The vast majority $(n=86,71 \%)$ were circumscribed. Of these lesions, 48 were classified as intraosseous and the remain as invasive. However, in case of suspected lesion, a needle biopsy should be performed to clarify diagnosis and assist with the decision on optimal management. 


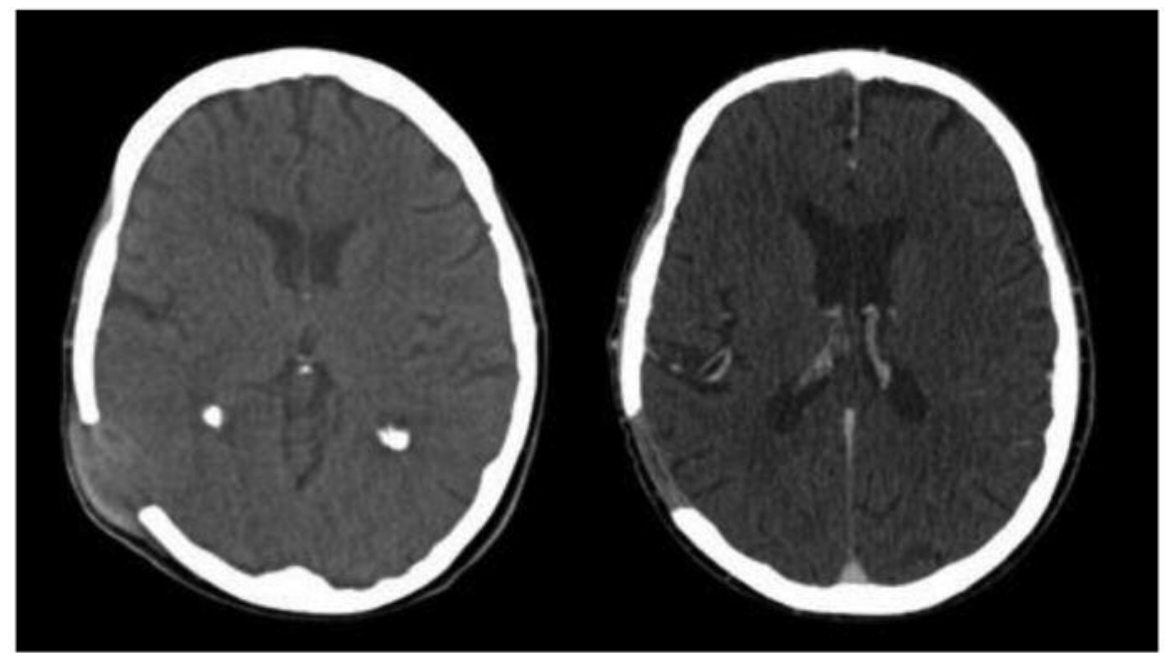

Figure 1. Computed tomography images of the right parietal bone metastasis before (left) and after (right) local radiation therapy.

At present, a universally accepted treatment approach cannot be determined. These studies have presented different treatment approaches, including single modality $(\mathrm{n}=138)(2,4,6,9,12$, 14-15, 20-21, 23-24, 28-29, 31-34), multidisciplinary options $(\mathrm{n}=54)(3-4,14,20-21,26-27)$, and palliative care/follow-up $(\mathrm{n}=120)(5,10,17-18,25,28-30,35)$. Of note, there are no treatment data in 27 cases. The collected studies do not show an absolute benefit of one treatment over the others as they are not case control studies and, therefore, are underpowered. We combined their results to add power to any tentative conclusion proposed by individual studies. However, no statistical value can be given to this analysis, thus the best treatment in cranial bone metastasis patients is still uncertain. Surgery alone with curative or palliative intent was proposed in 58 patients $(2,4-6,9-10,12$, 14-15, 17-18, 20-21, 23-25, 31-32, 34-35) and en-bloc resection was the preferred technique $(14,20-21)$. Systemic CHT, mainly using a platinum-based regimen, was especially provided to fit patients (36). In total, $126(37.2 \%)(3,4,14,20-21,26-30,33)$ patients underwent local RT, as exclusive (in 86 patients), adjuvant (in 34 patients) or neoadjuvant (in 6 patients) modality. Actually, adequate information on radiation technique, field size and margins, doses, fractionation schedules and beam energy were poorly documented and the little technical details available were heterogeneous. The treatment of choice was 3D conformal RT with conventional fractionation ( 2 Gy per fraction) up to 55 Gy (3, 29). Clinical experience with other RT techniques, such as stereotactic RT, was only limited to a retrospective analysis of 14 calvarial bone lesions (33). A 201-source 60Cobalt gamma knife radiosurgery system was used. To artificially extend the surface to target distance, a $5 \mathrm{~mm}$ bolus was applied over the treatment site in 7 cases. The median prescription margin dose was 15 Gy (range=13-24 Gy). The approach was feasible, safe and effective, also in those patients who had received prior RT.
In conclusion, cranial bone metastasis represents an extremely rare clinical scenario in patients with primary diagnosis of anal canal carcinoma. Maybe, its incidence is likely underreported due to no specific clinical presentation. Progress in oncological treatment, as well as modern imaging techniques routinely used in follow-up programs could slightly increase its diagnosis rates in the near future. MRI should be performed to better describe lesion characteristics. RT seems to be useful and could provide significant local control and positively influence patient's QoL. Surely, a multidisciplinary team should offer to patients the most appropriate treatment option, mainly according to primary tumor control, estimated survival, performance status and comorbidities.

\section{Conclusion}

Treatment for cranial bone metastatic anal cancer is anecdotal. Local RT and systemic CHT represent a safe and effective multimodal treatment option, with optimal local control and no severe toxicity.

\section{Conflicts of Interest}

All Authors declare no conflicts of interest regarding this study.

\section{Authors' Contributions}

IB prepared the literature search and wrote the manuscript under the close supervision of FDF. FDF guaranteed the integrity of entire study. DM designed the study preliminarily and contributed to data acquisition. $\mathrm{NB}, \mathrm{RC} 1$ and $\mathrm{VT}$ participated in the design and coordination of the case. IB, FDF, NB, RC1, RC2 DM, VT critically revised the manuscript and approved it. 
Table I. Case reports and case series for calvarial metastases.

\begin{tabular}{|c|c|c|c|c|c|c|c|c|}
\hline \multirow[b]{2}{*}{ Author } & \multicolumn{2}{|c|}{ Study } & \multirow[b]{2}{*}{ Patient } & \multirow[b]{2}{*}{$\begin{array}{l}\text { Primary } \\
\text { tumor }\end{array}$} & \multirow[b]{2}{*}{$\begin{array}{l}\text { Bone involved } \\
\text { (n lesions) }\end{array}$} & \multirow[b]{2}{*}{$\begin{array}{l}\text { Symptoms } \\
\text { (n cases) }\end{array}$} & \multirow[b]{2}{*}{ Treatment } & \multirow[b]{2}{*}{$\begin{array}{l}\text { Overall } \\
\text { survival }\end{array}$} \\
\hline & Type & Year & & & & & & \\
\hline $\begin{array}{l}\text { Constans } \\
\text { et al. (3) }\end{array}$ & Retrospective & 1981 & 14 & $\begin{array}{l}\text { Breast }(3 \mathrm{pz}) \\
\text { Thyroid }(3 \mathrm{pz}) \\
\text { Liver }(3 \mathrm{pz}) \\
\text { Kidney }(2 \mathrm{pz}) \\
\text { Colon }(1 \mathrm{pz}) \\
\text { Testicle }(1 \mathrm{pz}) \\
\text { Unknow }(1 \mathrm{pz})\end{array}$ & $\begin{array}{l}\text { Parietal (6) } \\
\text { Frontal (3) } \\
\text { Occipital (3) } \\
\text { Temporal (1) } \\
\text { Central (1) }\end{array}$ & $\begin{array}{c}\text { Asymptomatic (3) } \\
\text { Headache (3) } \\
\text { Neurologic deficit (3) } \\
\text { Seizures (2) } \\
\text { Increased intracranial } \\
\text { pressure (2) }\end{array}$ & $\begin{array}{l}S+\operatorname{adj} R T \\
(40-55 \mathrm{~Gy})\end{array}$ & $\begin{array}{c}\text { 3-36 } \\
\text { months }\end{array}$ \\
\hline $\begin{array}{l}\text { Nagamine } \\
\text { et al. (4) }\end{array}$ & Retrospective & 1985 & 11 & Thyroid & $\begin{array}{c}\text { Occipital (5) } \\
\text { Parieto-occipital (3) } \\
\text { Parietal (1) } \\
\text { Frontal (1) } \\
\text { Fronto-parietal (1) } \\
\text { Parito-occipital }\end{array}$ & $\begin{array}{l}\text { Swelling (11) } \\
\text { Headache (1) } \\
\text { Hemiparesis (1) } \\
\text { Disturbance of } \\
\text { consciousness (1) } \\
\text { Acute epidural } \\
\text { hematoma }\end{array}$ & $\begin{array}{c}\mathrm{S}+\operatorname{adj} \mathrm{RT}(4 \mathrm{pz}) \\
\mathrm{S}+\operatorname{adj} \text { CHT }(1 \mathrm{pz}) \\
\mathrm{S}+\operatorname{adj} \mathrm{RT}+\mathrm{CHT}(1 \mathrm{pz}) \\
\mathrm{RT}(1 \mathrm{pz}) \\
\mathrm{S}(4 \mathrm{pz}) \\
\mathrm{S}\end{array}$ & $\begin{array}{c}4.5 \text { years } \\
\text { (mean) }\end{array}$ \\
\hline $\begin{array}{l}\text { Kuratsu } \\
\text { et al. }(6)\end{array}$ & Case report & 1990 & 2 & Liver & $\begin{array}{c}\text { Occipital (1) } \\
\text { Frontal (1) }\end{array}$ & Painless mass (2) & S & $\begin{array}{l}6 \text { months } \\
1 \text { month }\end{array}$ \\
\hline $\begin{array}{l}\text { Sunita } \\
\text { et al. (7) }\end{array}$ & Case report & 1991 & 1 & Kidney & Frontal & Asymptomatic & - & - \\
\hline $\begin{array}{l}\text { Molina } \\
\text { et al. (8) }\end{array}$ & Case report & 1991 & 1 & Kidney & Occipital & Asymptomatic & - & 14 months \\
\hline $\begin{array}{l}\text { Nakao } \\
\text { et al. (9) }\end{array}$ & Case report & 1992 & 1 & Liver & Frontal & Palpable mass & S & 15 months \\
\hline $\begin{array}{l}\text { Nakagawa } \\
\text { et al. (10) }\end{array}$ & Case report & 1992 & 1 & Liver & Occipital & $\begin{array}{l}\text { Acute epidural } \\
\text { hematoma }\end{array}$ & S & 1 month \\
\hline $\begin{array}{l}\text { Cheah } \\
\text { et al. (11) }\end{array}$ & Case report & 1992 & 1 & Kidney & - & - & - & - \\
\hline $\begin{array}{l}\text { Yoshida } \\
\text { et al. (12) }\end{array}$ & Case report & 1993 & 1 & Liver & Frontal & Painfull mass & S & 8 month \\
\hline $\begin{array}{l}\text { Wahner- } \\
\text { Roedler } \\
\text { et al. (13) }\end{array}$ & Case report & 1997 & 1 & Kidney & Occipital & Asymptomatic & - & 15 months \\
\hline $\begin{array}{l}\text { Wecht } \\
\text { et al. (14) }\end{array}$ & Case series & 1997 & 7 & $\begin{array}{c}\text { Kidney (3 pz) } \\
\text { Melanoma (1 pz) } \\
\text { Lung (1 pz) } \\
\text { Other (2 pz) }\end{array}$ & - & - & $\begin{array}{c}\text { S (6 pz) } \\
\text { neo-adj RT + } \\
\text { CHT + S (1 pz) }\end{array}$ & $\begin{array}{c}20.6 \\
\text { months } \\
\text { (mean) }\end{array}$ \\
\hline $\begin{array}{l}\text { Koutnouyan } \\
\text { et al. (15) }\end{array}$ & Case report & 1998 & 1 & Kidney & Frontal & Asymptomatic & S & 9 months \\
\hline $\begin{array}{l}\text { Nitta } \\
\text { et al. (16) }\end{array}$ & Case report & 1999 & 1 & Kidney & Occipital & Painfull mass & - & - \\
\hline $\begin{array}{l}\text { Hayashi } \\
\text { et al. (17) }\end{array}$ & Case report & 2000 & 1 & Liver & Parietal & Acute epidural hematoma & $S$ & 2 months \\
\hline $\begin{array}{l}\text { McIver } \\
\text { et al. }(18)\end{array}$ & Case report & 2001 & 1 & Liver & Parietal & Acute epidural hematoma & $S$ & - \\
\hline $\begin{array}{l}\text { Yamamoto } \\
\text { et al. (19) }\end{array}$ & Case report & 2001 & 1 & Kidney & Frontal & Asymptomatic & - & - \\
\hline $\begin{array}{l}\text { Michael } \\
\text { et al. }(20)\end{array}$ & Retrospective & 2001 & $27 *$ & $\begin{array}{l}\text { Kidney }(13 \mathrm{pz}) \\
\text { Sarcoma }(6 \mathrm{pz}) \\
\text { Prostate }(4 \mathrm{pz}) \\
\text { Melanoma }(3 \mathrm{pz}) \\
\text { Other }(1 \mathrm{pz})\end{array}$ & $\begin{array}{c}\text { Fronto-parietal (5) } \\
\text { Frontal (3) } \\
\text { Occipital (2) } \\
\text { Parietal (2) } \\
\text { Parieto-occipital (1) }\end{array}$ & $\begin{array}{l}\text { Painless mass (11) } \\
\text { Painful mass (8) } \\
\text { Neurologic deficit (5) } \\
\text { Headache/blurred } \\
\text { vision (4) }\end{array}$ & $\begin{array}{c}\text { neo-adj CHT + S (12 pz) } \\
\text { neo-adj RT + S }(2 \mathrm{pz}) \\
\text { neo-adj RT + CHT } \\
+\mathrm{S}(3 \mathrm{pz}) \\
\mathrm{S}(8 \mathrm{pz}) \\
\text { Other }(2 \mathrm{pz})\end{array}$ & $\begin{array}{c}16.5 \\
\text { months } \\
\text { (median) }\end{array}$ \\
\hline
\end{tabular}


Table I. Continued

\begin{tabular}{|c|c|c|c|c|c|c|c|c|}
\hline \multirow[b]{2}{*}{ Author } & \multicolumn{2}{|c|}{ Study } & \multirow[b]{2}{*}{ Patient } & \multirow[b]{2}{*}{$\begin{array}{l}\text { Primary } \\
\text { tumor }\end{array}$} & \multirow[b]{2}{*}{$\begin{array}{l}\text { Bone involved } \\
\text { (n lesions) }\end{array}$} & \multirow[b]{2}{*}{$\begin{array}{l}\text { Symptoms } \\
\text { (n cases) }\end{array}$} & \multirow[b]{2}{*}{ Treatment } & \multirow[b]{2}{*}{$\begin{array}{l}\text { Overall } \\
\text { survival }\end{array}$} \\
\hline & Type & Year & & & & & & \\
\hline $\begin{array}{l}\text { Stark } \\
\text { et al. }(21)\end{array}$ & Retrospective & 2003 & $12 * *$ & $\begin{array}{c}\text { Breast }(4 \mathrm{pz}) \\
\text { Melanoma }(2 \mathrm{pz}) \\
\text { Colorectal }(1 \mathrm{pz}) \\
\text { Prostate }(1 \mathrm{pz}) \\
\text { Parotid }(1 \mathrm{pz}) \\
\text { Sarcoma }(3 \mathrm{pz})\end{array}$ & - & $\begin{array}{l}\text { Swelling (5) } \\
\text { Local pain (3) }\end{array}$ & $\begin{array}{c}\mathrm{S}(8 \mathrm{pz}) \\
\mathrm{S}+\operatorname{adj} \mathrm{RT}(4 \mathrm{pz})\end{array}$ & $\begin{array}{l}19.5 \\
\text { months } \\
\text { (mean) }\end{array}$ \\
\hline $\begin{array}{l}\text { Arana } \\
\text { et al. }(22)\end{array}$ & Retrospective & 2004 & 21 & - & $\begin{array}{l}\text { Parietal (15) } \\
\text { Frontal (13) } \\
\text { Occipital (3) }\end{array}$ & $\begin{array}{l}\text { Palpable mass (16) } \\
\text { Neurologic deficit (3) }\end{array}$ & - & - \\
\hline $\begin{array}{l}\text { Gaetani } \\
\text { et al. }(23)\end{array}$ & Case report & 2005 & 2 & Kidney & $\begin{array}{c}\text { Frontal (1) } \\
\text { Occipital (1) }\end{array}$ & Asymptomatic & $S$ & 9 months \\
\hline $\begin{array}{l}\text { Miyamoto } \\
\text { et al. }(24)\end{array}$ & Case report & 2007 & 1 & $\begin{array}{l}\text { Cholangio- } \\
\text { carcinoma }\end{array}$ & Occipital & Painfull mass & $\mathrm{S}$ & 6 months \\
\hline $\begin{array}{l}\text { Kanai } \\
\text { et al. }(25)\end{array}$ & Case report & 2008 & 1 & Liver & Parieto-occipital & Acute epidural hematoma & S & 3 weeks \\
\hline $\begin{array}{l}\text { Hong } \\
\text { et al. }(26)\end{array}$ & Case series & 2010 & 9 & $\begin{array}{l}\text { Breast }(2 \mathrm{pz}) \\
\text { Lung }(1 \mathrm{pz}) \\
\text { Kidney }(1 \mathrm{pz}) \\
\text { Other }(5 \mathrm{pz})\end{array}$ & $\begin{array}{l}\text { Parietal (3) } \\
\text { Occipital (3) } \\
\text { Frontal (2) } \\
\text { Temporal (1) }\end{array}$ & $\begin{array}{l}\text { Asymptmatic (3) } \\
\text { Painless mass (3) } \\
\text { Painful mass (3) }\end{array}$ & $S+\operatorname{adj} R T$ & $\begin{array}{l}5.8 \text { years } \\
(\text { mean })\end{array}$ \\
\hline $\begin{array}{l}\text { Chye } \\
\text { et al. (27) }\end{array}$ & Case report & 2011 & 1 & Liver & Temporal & Subdural hematoma & $\begin{array}{c}\mathrm{S}+\text { Chemoembolization } \\
+ \text { adj RT }\end{array}$ & - \\
\hline $\begin{array}{l}\text { Mitsuya } \\
\text { et al. }(28)\end{array}$ & Retrospective & 2011 &  & $\begin{array}{l}\text { Breast }(96 \mathrm{pz}) \\
\text { Lung }(25 \mathrm{pz}) \\
\text { Prostate }(11 \mathrm{pz}) \\
\text { Lymphoma }(9 \mathrm{pz}) \\
\text { Head and } \\
\text { neck }(1 \mathrm{pz}) \\
\text { Other }(34 \mathrm{pz})\end{array}$ & - & $\begin{array}{c}\text { Pain (5) } \\
\text { Cosmetic problem (3) } \\
\text { Meningeal irritation (3) } \\
\text { Disorientation (3) }\end{array}$ & $\begin{array}{c}\text { RT (63 pz) } \\
\text { Follow-up (112 pz) }\end{array}$ & - \\
\hline $\begin{array}{l}\text { Fujimoto } \\
\text { et al. }(29)\end{array}$ & Case report & 2013 & 2 & $\begin{array}{l}\text { Cholangio- } \\
\text { carcinoma }\end{array}$ & $\begin{array}{l}\text { Parietal (2) } \\
\text { Temporal (1) }\end{array}$ & $\begin{array}{c}\text { Pailfull mass (2) } \\
\text { Neurologic deficit (1) }\end{array}$ & $\begin{array}{c}\text { SRT }(1 \mathrm{pz}) \\
\text { RT }(30 \mathrm{~Gy})+\mathrm{CHT} \\
+ \text { SRT }(1 \mathrm{pz})\end{array}$ & $\begin{array}{l}12 \text { months } \\
4 \text { years }\end{array}$ \\
\hline $\begin{array}{l}\text { Kumar } \\
\text { et al. }(30)\end{array}$ & Case report & 2014 & 1 & Ovarian & Parietal & Acute epidural hematoma & $S+\operatorname{adj} R T$ & \\
\hline $\begin{array}{l}\text { Cecchi } \\
\text { et al. }(31)\end{array}$ & Case report & 2014 & 1 & Endometrium & Temporo-parietal & Painless mass & $\mathrm{S}$ & 4 months \\
\hline $\begin{array}{l}\text { Guo } \\
\text { et al. (32) }\end{array}$ & Case report & 2014 & 1 & Liver & Parieto-occipital & Painless mass & $\mathrm{S}$ & 18 months \\
\hline $\begin{array}{l}\text { Kotecha } \\
\text { et al. }(33)\end{array}$ & Case series & 2014 & $21 * * * *$ & $\begin{array}{c}\text { Breast }(5 \mathrm{pz}) \\
\text { Kidney }(3 \mathrm{pz}) \\
\text { Sarcoma }(3 \mathrm{pz}) \\
\text { Liver }(2 \mathrm{pz}) \\
\text { Head and } \\
\text { neck }(2 \mathrm{pz}) \\
\text { Other }(6 \mathrm{pz})\end{array}$ & - & - & GKS RT (13-24Gy) & $\begin{array}{c}35.9 \\
\text { months } \\
\text { (median) }\end{array}$ \\
\hline $\begin{array}{l}\text { Chen } \\
\text { et al. (34) }\end{array}$ & Case report & 2015 & 1 & Liver & - & - & $S$ & - \\
\hline $\begin{array}{l}\text { Kim } \\
\text { et al. }(35)\end{array}$ & Case report & 2016 & 1 & Liver & Parieto-occipital & $\begin{array}{l}\text { Acute epidural } \\
\text { hematoma }\end{array}$ & $S$ & 4 months \\
\hline $\begin{array}{l}\text { Ozgiray } \\
\text { et al. }(2)\end{array}$ & Retrospective & 2016 & 15 & $\begin{array}{l}\text { Thyroid }(3 \mathrm{pz}) \\
\text { Sarcoma }(2 \mathrm{pz}) \\
\text { Breast }(1 \mathrm{pz}) \\
\text { Cholangio- } \\
\text { carcinoma }(1 \mathrm{pz}) \\
\text { Colon }(1 \mathrm{pz}) \\
\text { Other }(7 \mathrm{pz})\end{array}$ & $\begin{array}{c}\text { Frontal (9) } \\
\text { Parietal (6) } \\
\text { Occipital (2) } \\
\text { Cerebellar (1) }\end{array}$ & $\begin{array}{c}\text { Swelling (10) } \\
\text { Local pain (5) } \\
\text { Neurologic deficit (1) } \\
\text { Skin ulceration (1) }\end{array}$ & $S$ & - \\
\hline
\end{tabular}

*The bones involved were specified only for dural sinus calvarial metastases; $* *$ Include 5 patients with skull-base metastases; $* * *$ Include 43 patients with associated skull-base metastases; ****Include 12 patients with skull-base metastases. N: Number; S: surgery; adj: adjuvant; RT: radiotherapy; Gy: Gray; CHT: chemotherapy; neo-adj: neo-adjuvant; GKS: gamma knife surgery. 


\section{References}

1 Kakhki VR, Anvari K, Sadeghi R, Mahmoudian AS and Torabian-Kakhki M: Pattern and distribution of bone metastases in common malignant tumors. Nucl Med Rev Cent East Eur 16(2): 66-69, 2013. PMID: 24068635. DOI: 10.5603/NMR. 2013.0037

2 Ozgiray E, Perumal K, Cinar C, Caliskan Ke, Ertan Y, Yurtseven, Oktar N, Ovul I and Oner: Management of calvarial tumors: a retrospective analysis and literature review. Turk Neurosurg 26: 690-698, 2016. PMID: 27337230. DOI: 10.5137/ 1019-5149.JTN.12537-15.0.

3 Constans JP and Donzelli R: Surgical features of cranial metastases. Surg Neurol 15(1): 35-38, 1981. PMID: 7256522.

4 Nagamine Y, Suzuki J, Katakura R, Yoshimoto T, Matoba N and Takaya K: Skull metastasis of thyroid carcinoma. Study of 12 cases. J Neurosurg 63(4): 526-531, 1985. PMID: 4032016. DOI: 10.3171/jns.1985.63.4.0526

5 Anegawa S, Hirohata S, Tokutomi $\mathrm{T}$ and Kuramoto $\mathrm{S}$ : Spontaneous epidural hematoma secondary to dural metastasis from an ovarian carcinoma - case report. Neurol Med Chir (Tokyo) 29(9): 854-856, 1989. PMID: 2480548.

6 Kuratsu J, Murakami M, Uemura S and Ushio Y: Brain and skull metastases of hepatic or pancreatic cancer-report of six cases. Neurol Med Chir (Tokyo) 30: 476-482, 1990. PMID: 1701858.

7 Sunita, Kapila K, Singhal RM and Verma K: Fine needle aspiration diagnosis of an unusual presentation of metastatic renal cell carcinoma. Acta Cytol 35: 260-261, 1991. PMID: 1701858.

8 Molina M, Ortega G, de Paco M and Seller G: Solitary cranial metastasis as an initial manifestation of renal carcinoma. Rev Clin Esp 189: 196-197, 1991. PMID: 1745816.

9 Nakao N, Kubo K and Moriwaki H: Cranial metastasis of hepatocellular carcinoma associated with chronic epidural hematoma-case report. Neurol Med Chir 32(2): 100-103, 1992. PMID: 1376859.

10 Nakagawa Y, Yoshino E, Suzuki K, Tatebe A and Andachi H: Spontaneous epidural hematoma from a hepatocellular carcinoma metastasis to the skull-case report. Neurol Med Chir (Tokyo) 32(5): 300-302, 1992. PMID: 1378949.

11 Cheah PL, Looi LM and Lin HP: Clear cell sarcoma of kidney: a clinicopathological study of eight cases from Malaysia. Histopathology 21: 365-369, 1992. PMID: 1328018.

12 Yoshida D, Chen MN, Awaya S and Nakazawa S: Cranial metastasis of hepatocellular carcinoma in a female-case report. Neurol Med Chir (Tokyo) 33(12): 839-844, 1993.

13 Wahner-Roedler L and Sebo T: Renal cell carcinoma; diagnosis based on metastatic manifestations (case report). Mayo Clin Proc 72: 935-941, 1997. PMID: 7512232.

14 Wecht DA and Sawaya R: Lesions of the calvaria: Surgical experience with 42 patients. Ann Surg Oncol 4(1): 28-36, 1997. PMID: 8985515.

15 Koutnouyan HA, Rumore GJ and Kahn JM: Skull metastasis from renal cell carcinoma. Case report and literature review. Ann Otol Rhinol Laryngol 107: 598-602, 1998. PMID: 9682856. DOI: $10.1177 / 000348949810700710$

16 Nitta T, Kataoka H and Hirai O: Metastatic calvarial tumor: Report of 3 cases. No Shinkei Geka 27: 347-352, 1999. PMID: 10347850

17 Hayashi K, Matsuo T, Kurihara M, Daikoku M, Kitange G and Shibata S: Skull metastasis of hepatocellular carcinoma associated with acute epidural hematoma: A case report. Surg Neurol 53(4): 379-382, 2000. PMID: 10825524.

18 McIver JI, Scheithauer BW, Rydberg CH and Atkinson JL: Metastatic hepatocellular carcinoma presenting as epidural hematoma: case report. Neurosurgery 49(2): 447-449, 2001. PMID: 11504122.

19 Yamamoto AJ, Zhuang $\mathrm{H}$ and Alavi A: Detection of cranial metastases by F-18 FDG positron emission tomography. Clin Nucl Med 26: 402-404, 2001. PMID: 11317019.

20 Michael CB, Gokaslan ZL, DeMonte F, McCutcheon IE, Sawaya $\mathrm{R}$ and Lang FF: Surgical resection of calvarial metastases overlying dural sinuses. Neurosurgery 48: 745-754, 2001. PMID: 11322434.

21 Stark AM, Eichmann T and Mehdorn HM: Skull metastases: Clinical features, differential diagnosis, and review of the literature. Surg Neurol 60(3): 219-225, 2003. PMID: 12922038.

22 Arana E, Marti-Bonmati L, Bautista D and Paredes R: Qualitative diagnosis of calvarial metastasis by neural network and logistic regression. Acad Radiol 11: 45-52, 2004. PMID: 14746401.

23 Gaetani P, Di Ieva A, Colombo P, Tancioni F, Aimar E, Debernardi A and Baena RR: Calvarial metastases as clinical presentation of renal cell carcinoma: Report of two cases and review of the literature. Clin Neurol Neurosurg 107(4): 329-333, 2005. PMID: 15885394. DOI: 10.1016/j.clineuro.2004.07.017

24 Miyamoto J, Tatsuzawa K, Sasajima H and Mineura K: Metastatic skull tumor from cholangiocarcinoma. Case report. Neurol Med Chir (Tokyo) 47(3): 132-135, 2007. PMID: 17384497.

25 Kanai R, Kubota H, Terada T, Hata T, Tawaraya E and Fujii K: Spontaneous epidural hematoma due to skull metastasis of hepatocellular carcinoma. J Clin Neurosci 16(1): 137-140, 2009. PMID: 19013817. DOI: 10.1016/j.jocn.2008.02.020

26 Hong B, Hermann EJ, Klein R, Krauss JK and Nakamura M: Surgical resection of osteolytic calvarial lesions: Clinicopathological features. Clin Neurol Neurosurg 112(10): 865-869, 2010. PMID: 20685034. DOI: 10.1016/j.clineuro. 2010.07.010

27 Chye CL, Lin KH, Ou CH, Sun CK, Chang IW and Liang CL: Acute spontaneous subdural hematoma caused by skull metastasis of hepatocellular carcinoma: case report. BMC Surg 15: 60, 2015. PMID: 25958386. DOI: 10.1186/s12893-015-0045-x

28 Mitsuya K, Nakasu Y, Horiguchi S, Harada H, Nishimura T, Yuen S, Asakura K and Endo M: Metastatic skull tumors: MRI features and a new conventional classification. J Neurooncol 104(1): 239-245, 2011. PMID: 21110218. DOI: 10.1007/s11060010-0465-5

29 Fujimoto K, Kuroda J, Makino K, Hasegawa Y and Kuratsu J: Skull metastasis from intrahepatic cholangiocarcinoma: Report of 3 cases and review of the literature. Neurol Med Chir (Tokyo) 53: 717-721, 2013. PMID: 24077267.

30 Kumar PM and Manisha M: Epidural hematoma secondary to solitary skull metastasis from an ovarian carcinoma. Asian J Neurosurg 9(2): 112-114, 2014. PMID: 25126131. DOI: 10.4103/1793-5482.136729

31 Cecchi PC, Kluge R and Schwarz A: Calvarial metastasis from endometrial carcinoma: Case report and review of the literature. Asian J Neurosurg 9: 242-243, 2014. PMID: 25685234. DOI: 10.4103/1793-5482.146648

32 Guo X, Yin J and Jiang Y: Solitary skull metastasis as the first symptom of hepatocellular carcinoma: case report and literature review. Neuropsychiatr Dis Treat 10: 681-686, 2014. PMID: 24812512. DOI: $10.2147 /$ NDT.S58059 
33 Kotecha R, Angelov L, Barnett GH, Reddy CA, Suh JH, Murphy ES, Neyman G and Chao ST: Calvarial and skull base metastases: expanding the clinical utility of gamma knife surgery. J Neurosurg 121: 91-101, 2014. PMID: 25434942. DOI: 10.3171/2014.7.GKS141272

34 Chen R, Gao Z, Wu X, Campbell JL, Zhang P and Chen B: Hepatocellular carcinoma presenting as thoracic spinal canal metastasis with no clinical primary foci: a report of a rare case and review of the literature. Oncol Lett 10: 2333-2336, 2015. PMID: 26622846. DOI: 10.3892/ol.2015.3569

35 Kim YS, Moon KS, Lee KH, Jung TY, Jang WY, Kim IY and Jung S: Spontaneous acute epidural hematoma developed due to skull metastasis of hepatocelluar carcinoma: a case report and review of the literature. Oncol Lett 11: 741-744, 2016. PMID: 26870277. DOI: $10.3892 / o 1.2015 .3947$

36 Harrison RA, Nam JY, Weathers S P and De Monte F: Intracranial dural, calvarial, and skull base metastases. In: Handbook of Clinical Neurology, vol. 149, Elsevier BV, pp. 323, 2012. DOI: 10.1016/B978-0-12-811161-1.00014-1
37 Marchetti C, Pisano C, Mangili G, Lorusso D, Panici PB, Silvestro G, Candiani M, Greggi S, Perniola G, Di Maio M and Pignata S: Use of adjuvant therapy in patients with FIGO stage III endometrial carcinoma: a multicenter retrospective study. Oncology 81(2): 104-112, 2011. PMID: 21986449 DOI: $10.1159 / 000331677$

38 Weiner AB, Cortes-Mateus S, De Luis E and Duran I: Dural metastases in advanced prostate cancer: a case report and review of the literature. Curr Urol 7: 166-168, 2014. PMID: 24917781. DOI: $10.1159 / 000343558$

39 Amaral L, Chiurciu M, Almeida JR, Ferreira NF, Mendonca R and Lima SS: MR imaging for evaluation of lesions of the cranial vault. Arq Neuropsiquiatr 61(3A): 521-532, 2003. PMID: 14513152.

Received April 3, 2019

Revised May 9, 2019

Accepted May 10, 2019 